\title{
EDITORIAL OPEN What if it was easier to prevent schizophrenia than to treat it?
}

npj Schizophrenia (2017)3:9; doi:10.1038/s41537-

017-0012-x

Neural tube defects occur when the brain or the spinal cord fails to close early in embryonic development. While genetic polymorphisms affecting folate metabolism suggest that only certain individuals may be at increased risk for neural tube defects, ${ }^{1}$ since 1992 all women of childbearing age have been recommended to consume daily folic acid. ${ }^{2}$ This proved so effective that wheat products are now fortified as well. ${ }^{2}$ Our ability to prevent neural tube defects, with only a vitamin, demonstrates that we must now consider the idea that we can preclude other neurodevelopmental disorders as well.

Schizophrenia is preceded by a long period of disease progression prior to the onset of symptoms. How best to study a disease prior to diagnosis? Classically, physicians and scientists have done this by tracking high-risk individuals for decades, waiting for symptom onset to occur in a small subset of their cohort. This is both time-consuming and inefficient. With the Nobel Prize winning discovery by Shinya Yamanaka in 2007 , it is now possible to reprogram human-induced pluripotent stem cells (hiPSCs) from patient cells; ${ }^{3}$ these hiPSCs have the ability to differentiate into all cell types found in the body. ${ }^{4}$ Suddenly, scientists gained the ability to generate stem cells from every person on the planet, providing the opportunity to study disease processes in patient-derived cells cultured in a laboratory dish.

Current hiPSC differentiation strategies yield neurons that mostly resemble the fetal brain cells (Brennand et al. 2015 Molecular Psychiatry; Mariani et al. 2012 PNAS; Nicholas et al. 2013, Cell Stem Cell; Pasca et al., Nature Methods 2015), making them a better tool for the study of the molecular aspects of disease predisposition, rather than the disease-state itself. For example, hiPSC-based studies of late onset neurodegenerative diseases such as Parkinson's Disease, ${ }^{5-8}$ Alzheimer's Disease ${ }^{9,} 10$ and amyotrophic lateral sclerosis ${ }^{11}$ have failed to recapitulate the severe neuronal loss observed in human disease. Using hiPSCs, we and others have found that schizophrenia hiPSC-derived neural progenitor cells show evidence of aberrant migration, ${ }^{12}$ deficits associated with adherens junctions and polarity, ${ }^{13}$ increased oxidative stress $^{12,14,15}$ and perturbed responses to environmental stressors; ${ }^{16}$ while schizophrenia hiPSC-derived neurons exhibit decreased neurite number, ${ }^{17}$ reduced synaptic maturation ${ }^{14,}$ 17-19 and synaptic activity, ${ }^{18}, 19$ and blunted activity-dependent response. ${ }^{20}$ These in vitro deficits may reflect processes underlying disease predisposition in patients. Consistent with this, we recently reported unbiased hiPSC-based evidence ${ }^{21}$ that was convergent with novel human genetics-based analyses, ${ }^{22}$ suggesting that microRNA-9 may partially contribute to genetic risk for schizophrenia in a subset of patients.

While the potential of hiPSC-based models to establish a personalized medicine approach to the treatment of schizophrenia-one drug screen per genotype-has been widely discussed, ${ }^{23}$ here I posit that the first hiPSC-based screens may instead identify drugs more suitable for disease prevention. It may ultimately prove easier to apply hiPSC-based models towards the prevention, rather than treatment, of schizophrenia.

\section{ACKNOWLEDGEMENTS}

Kristen Brennand is a New York Stem Cell Foundation-Robertson Investigator. Her laboratory is supported in part by a National Institute of Health $(\mathrm{NIH})$ grant R01 MH101454, New York Stem Cell Foundation and the Brain and Behavior Research Foundation.

\section{COMPETING INTERESTS}

The author declares no competing interests.

Kristen J. Brennand (iD) ${ }^{1,2}$

${ }^{1}$ Departments of Psychiatry and Neuroscience, Icahn School of Medicine at Mount Sinai, 1425 Madison Avenue, New York, NY 10029, USA and ${ }^{2}$ Friedman Brain Institute, Icahn School of Medicine at Mount Sinai, 1425 Madison Avenue, New York, NY 10029, USA Correspondence: Kristen J. Brennand (kristen.brennand@mssm.edu)

\section{REFERENCES}

1. Molloy, A. M., Brody, L. C., Mills, J. L., Scott, J. M. \& Kirke, P. N. The search for genetic polymorphisms in the homocysteine/folate pathway that contribute to the etiology of human neural tube defects. Birth Defects Res. A Clin. Mol. Teratol. 85, 285-294 (2009).

2. Crider, K. S., Bailey, L. B. \& Berry, R. J. Folic acid food fortification-its history, effect, concerns, and future directions. Nutrients 3, 370-384 (2011).

3. Takahashi, K. et al. Induction of pluripotent stem cells from adult human fibroblasts by defined factors. Cell 131, 861-872 (2007).

4. Mertens, J., Marchetto, M. C., Bardy, C. \& Gage, F. H. Evaluating cell reprogramming, differentiation and conversion technologies in neuroscience. Nat. Rev. Neurosci. 17, 424-437 (2016).

5. Chung, C. Y. et al. Identification and rescue of alpha-synuclein toxicity in parkinson patient-derived neurons. Science 342, 983-987 (2013).

6. Nguyen, H. N. et al. LRRK2 mutant iPSC-derived DA neurons demonstrate increased susceptibility to oxidative stress. Cell Stem Cell 8, 267-280 (2011).

7. Byers, B. et al. SNCA triplication parkinson's patient's iPSC-derived DA neurons accumulate alpha-synuclein and are susceptible to oxidative stress. PLoS One 6, e26159 (2011).

8. Liu, G. H. et al. Progressive degeneration of human neural stem cells caused by pathogenic LRRK2. Nature 491, 603-607 (2012).

9. Israel, M. A. et al. Probing sporadic and familial Alzheimer's disease using induced pluripotent stem cells. Nature 482, 216-220 (2012).

10. Kondo, T. et al. Modeling alzheimer's disease with iPSCs reveals stress phenotypes associated with intracellular abeta and differential drug responsiveness. Cell Stem Cell 12, 487-496 (2013).

11. Sareen, D. et al. Targeting RNA foci in iPSC-derived motor neurons from ALS patients with a C9ORF72 repeat expansion. Sci. Transl. Med. 5, 208 ra149 (2013).

12. Brennand, K. J. et al. Phenotypic differences in hiPSC NPCs derived from patients with schizophrenia. Mol. Psychiatry 20, 361-368 (2014).

13. Yoon, K. J. et al. Modeling a genetic risk for schizophrenia in iPSCs and mice reveals neural stem cell deficits associated with adherens junctions and polarity. Cell Stem Cell 15, 79-91 (2014).

14. Robicsek, O. et al. Abnormal neuronal differentiation and mitochondrial dysfunction in hair follicle-derived induced pluripotent stem cells of schizophrenia patients. Mol. Psychiatry 18, 1067-1076 (2013).

15. Paulsen, B. D. et al. Altered oxygen metabolism associated to neurogenesis of induced pluripotent stem cells derived from a schizophrenic patient. Cell Transplant. 21, 1547-1559 (2011). 
16. Hashimoto-Torii, K. et al. Roles of heat shock factor 1 in neuronal response to fetal environmental risks and its relevance to brain disorders. Neuron 82, 560-572 (2014).

17. Brennand, K. J. et al. Modelling schizophrenia using human induced pluripotent stem cells. Nature 473, 221-225 (2011).

18. Yu, D. X. et al. Modeling hippocampal neurogenesis using human pluripotent stem cells. Stem Cell Rep. 2, 295-310 (2014).

19. Wen, Z. et al. Synaptic dysregulation in a human iPS cell model of mental disorders. Nature 515, 414-418 (2014).

20. Roussos, P., Guennewig, B., Kaczorowski, D. C., Barry, G. \& Brennand, K. J. Activitydependent changes in gene expression in schizophrenia human-induced pluripotent stem cell neurons. JAMA Psychiatry 73, 1180-1188 (2016).

21. Topol, A. et al. Dysregulation of miRNA-9 in a subset of schizophrenia patientderived neural progenitor cells. Cell Rep. 15, 1024-1036 (2016).

22. Hauberg, M. E. et al. Analyzing the role of MicroRNAs in schizophrenia in the context of common genetic risk variants. JAMA Psychiatry 73, 369-377 (2016).
23. Panchision, D. M. Concise review: progress and challenges in using human stem cells for biological and therapeutics discovery: neuropsychiatric disorders. Stem Cells 34, 523-536 (2016).

This work is licensed under a Creative Commons Attribution 4.0 International License. The images or other third party material in this article are included in the article's Creative Commons license, unless indicated otherwise in the credit line; if the material is not included under the Creative Commons license, users will need to obtain permission from the license holder to reproduce the material. To view a copy of this license, visit http://creativecommons.org/licenses/by/ $4.0 /$

(c) The Author(s) 2017 